\title{
Group of type III bursts and its associated spikes
}

\author{
S. J. Wang, Y. H. Yan, and Q. J. Fu \\ National Astronomical Observatories, Chinese Academy of Sciences, Beijing 100012, PR China \\ Received 6 March 2001 / Accepted 2 May 2001

\begin{abstract}
A group of type III bursts in solar radio bursts, observed with the 2.6-3.8 GHz spectrometer of the Beijing Astronomical Observatory on April 15 1998, is selected and analyzed. The type III bursts are associated with a large number of spikes, which have been recorded by the spectrometer at high temporal resolution $(8 \mathrm{~ms})$. We report the observational characteristics of the type III bursts and the corresponding spikes including duration, frequency bandwidth, drift rate and quasi-periodicity. Afterwards we discuss the space scale, the brightness temperature and the magnetic field strength at the emission source region. Finally we investigate the mechanism which would cause the quasi-periodicity of the type III bursts and the short time scale of the spikes theoretically. It is suggested that the type III bursts and the spikes differ clearly between their source regions in the space scale, the brightness temperature, the magnetic field strength and the magnetic structure.
\end{abstract}

Key words. Sun: radio radiation - Sun: corona

\section{Introduction}

Radio spectrometers with high temporal resolution and high spectral resolution have been developed by the solar radio astronomy community of China (Beijing Astronomical Observatory (BAO), Purple Mountain Observatory (PMO), Yunnan Astronomical Observatory (YAO), Nanjing University (NJU)) (Fu et al. 1995). Those in $1.0-2.0 \mathrm{GHz}, 2.6-3.8 \mathrm{GHz}, 5.2-7.6 \mathrm{GHz}$ bands have been in operation since the end of January 1994, September 1996 and July 1999, respectively. Many radio spikes, type III bursts and other faint events have been found by the spectrometers including: microwave millisecond spikes in the range of $2.6-3.8 \mathrm{GHz}$ on 2 November 1997 (Xie et al. 2000), 50 microwave type III bursts in the range of 1.0-2.0 GHz during January-April 1998 (Wang et al. 1999), drifting pulsation structures in the range of $1.0-2.0 \mathrm{GHz}$ and $5.2-7.6 \mathrm{GHz}$ on July $10-14$, 2000 (Karlický et al. 2001), U bursts and reverse-slope (RS)-drifting bursts on July 10, 2000 (Wang et al. 2001) and so on.

Radio spikes are often associated in time both with type III bursts and with hard X-ray bursts. All of them may reflect some information about the energetic electrons in solar corona, especially type III bursts, being well-known as the signatures of energetic electron beams in coronal plasma (Benz \& Kane 1986). The plasma density of spike sources indicated that the sources are close to or in the energy release region and located in a tiny

Send offprint requests to: S. J. Wang,

e-mail: wsj@sun10.bao.ac.cn or wsj888@263.net
Table 1. The periods of all channels (freq.).

\begin{tabular}{|c|c|c|c|c|c|}
\hline Left Polar. & & & Right Polar. & & \\
\hline Ch.(9-119) & $\tau$ & error & Ch.(129-239) & $\tau$ & error \\
\hline$(2.7-3.8 \mathrm{GHz})$ & $(\mathrm{s})$ & $(\mathrm{s})$ & $(2.7-3.8 \mathrm{GHz})$ & (s) & $(\mathrm{s})$ \\
\hline $9-26$ & 4.08 & 0.24 & $129-132$ & 4.08 & 0.21 \\
\hline $27-31$ & 4.66 & 0.21 & $133-151$ & 4.66 & 0.29 \\
\hline $32-38$ & 3.62 & 0.17 & $152-169$ & 4.08 & 0.23 \\
\hline $39-45$ & 3.26 & 0.12 & $170-181$ & 3.26 & 0.15 \\
\hline $46-96$ & 5.43 & 0.36 & $182-184$ & 5.43 & 0.35 \\
\hline $97-111$ & 3.62 & 0.20 & $185-195$ & 4.08 & 0.22 \\
\hline $112-116$ & 4.08 & 0.20 & $196-239$ & 5.43 & 0.38 \\
\hline $117-119$ & 4.66 & 0.26 & & & \\
\hline Average: & 4.60 & 0.27 & & 4.66 & 0.29 \\
\hline
\end{tabular}

volume, so the spikes associated with the type III bursts were regarded to be the fragmentation of an energy release process in a solar flare. Therefore type III bursts and HXR bursts were regarded to be the integrated effects of many smaller fractional events (elementary flare events) (Benz 1985). However, Fleǐshman \& Mel'nikov (1998) recently suggested that the radio spikes appear not to be due to the fragmentation of a primary energy release, but rather to the "secondary" fragmentation, i.e. due to the presence of sufficiently strong magnetic field inhomogeneities in a coronal loop, although these inhomogeneities themselves seem to directly result from the primary energy release.

In Sect. 2 of this paper, we report a group of type III bursts and its associated spikes recorded on April 15, 1998 at 2.7-3.8 GHz. In Sect. 3 the parameters of emission sources including the space scale, the brightness 
Table 2. Properties of duration, frequency bandwidth, frequency drift rate and central frequency of each type III burst, and the spatial range of the related source.

\begin{tabular}{cccccc}
\hline \hline No & $\Delta t$ & $\begin{array}{c}\Delta f \\
(\mathrm{GHz})\end{array}$ & $\begin{array}{c}\text { Prift rate } \\
\left(\mathrm{GHz} \mathrm{s}^{-1}\right)\end{array}$ & $\begin{array}{c}f_{0} \\
(\mathrm{GHz})\end{array}$ & $\begin{array}{c}\Delta h \\
\left(\times 10^{4} \mathrm{~km}\right)\end{array}$ \\
\hline $07: 59 ?(\mathrm{UT})$ & $(\mathrm{s})$ & 0.26 & 0.45 & 3.65 & 0.11 \\
$: 29-: 32$ & 1.8 & 0.26 & -0.65 & 3.23 & 0.13 \\
$: 35-: 37$ & 0.4 & 0.26 & 0.53 & 3.28 & 0.31 \\
$: 37-: 39$ & 1.2 & 0.64 & -0.53 & 3.66 & 0.11 \\
$: 42-: 44$ & 0.4 & 0.25 & 0.63 & 3.01 & 0.20 \\
$: 45-: 49$ & 0.4 & 0.36 & 0.90 & 0.20 \\
$: 51-: 52$ & 0.4 & 0.43 & -1.07 & 3.42 & 0.10 \\
$: 52-: 53$ & 0.4 & 0.24 & 0.60 & 3.67 & \\
\hline \multicolumn{7}{c}{ Right } & Polar. & & \\
\hline No & $\Delta t$ & $\Delta f$ & drift rate & $f_{0}$ & $\Delta h$ \\
$07: 59 ?(\mathrm{UT})$ & $(\mathrm{s})$ & $(\mathrm{GHz})$ & $\left(\mathrm{GHz} \mathrm{s}^{-1}\right)$ & $(\mathrm{GHz})$ & $\left(\times 10^{4} \mathrm{~km}\right)$ \\
\hline$: 29-: 32$ & 0.8 & 0.42 & 0.53 & 3.57 & 0.18 \\
$: 35-: 37$ & 0.2 & 0.36 & -1.80 & 2.87 & 0.21 \\
$: 37-: 39$ & 1.4 & 0.59 & -0.42 & 3.31 & 0.28 \\
$: 42-: 44$ & 0.4 & 0.25 & 0.63 & 3.66 & 0.11 \\
$: 45-: 49$ & 0.4 & 0.55 & 1.38 & 3.20 & 0.28 \\
$: 51-: 52$ & 0.4 & 0.61 & -1.53 & 3.30 & 0.29 \\
$: 52-: 53$ & 0.2 & 0.32 & 1.68 & 3.63 & 0.14 \\
\hline
\end{tabular}

temperature and the magnetic field strength are determined according to reasonable assumptions. We then discuss the quasi-periodicity of the type III bursts and the short time scale of the spikes using the magnetic reconnection mode and the tearing instability mode, respectively, in Sect. 4. The results suggest that the type III bursts and the spikes differ clearly in their source regions in the space scale, the brightness temperature, the strength and structure of magnetic field. In Sect. 5 some conclusions are given.

\section{Observational properties}

\subsection{Instruments}

The radio observations were carried out with the 2.6$3.8 \mathrm{GHz}$ spectrometer of BAO. The band of the spectrometer $(2.6-3.8 \mathrm{GHz})$ is divided into 120 channels and each channel has a bandwidth of $10 \mathrm{MHz}$. The channels 1-120 are for the left polarization (LP) and the channels 121240 for the right polarization (RP). Data are recored in terms of circular polarization and total flux spectra simultaneously. The instrument has a time resolution of $8 \mathrm{~ms}$. The integration at $0.2 \mathrm{~s}$ is also recorded. The sensitivity is better than $2 \%$ of the quiet Sun level and the accuracy of the polarization degree is better than 5-10\% (Fu et al. 1995).

\subsection{Type III bursts}

On 15 April 1998, a group of quasi-periodic type III bursts was detected by the $2.6-3.8 \mathrm{GHz}$ radio spectrometer of $\mathrm{BAO}$ at $0.2 \mathrm{~s}$ time resolution at 07:59:25-07:59:57 UT. The data of the first ten channels $(2.6-2.69 \mathrm{GHz})$ were not

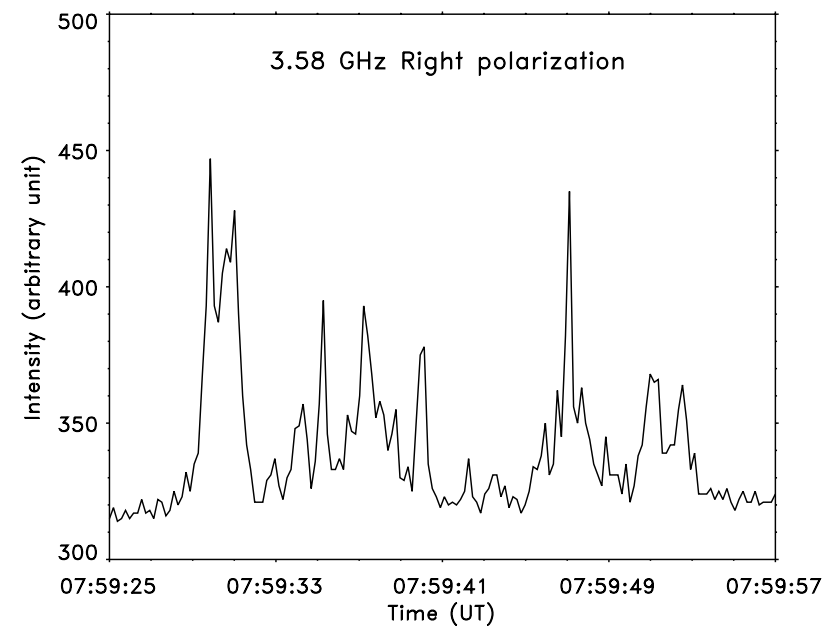

Fig. 1. Single-frequency curve of radio bursts at $3.58 \mathrm{GHz}$ recorded by BAO spectrometer on 15 April, 1998. Spikes were superimposed on the smoother, impulsive emission.

so good, so they are not selected. The time profile (for the right-polarized component only) of bursts at $3.58 \mathrm{GHz}$ is shown in Fig. 1. After subtracting the background noise, the gray-scale plots of the group of type III bursts are shown in Fig. 2.

As shown in Fig. 2, this event contained about seven quasi-periodic type III bursts. After FFT analysis, the periods of all channels are listed in Table 1 . The average periods were $4.60 \mathrm{~s}$ for LCP and $4.66 \mathrm{~s}$ for RCP. The properties of duration, frequency bandwidth, frequency drift rate and central frequency of each type III burst, and also the spatial height of the related source are summarized in Table 2. Statistically the average duration of a single type III burst was about $0.6 \mathrm{~s}$, the frequency bandwidth $12 \%$ of the center frequency, and the frequency drift rate $900 \mathrm{MHz} / \mathrm{s}$. Both reverse (positive) drift as well as normal (negative) drift occured in the type III bursts. There was no predominance of the positive or the negative sign. The flux density of RP was stronger than that of LP. The mean polarization degree was about $70 \% \mathrm{RP}$. The total flux density $(\mathrm{LP}+\mathrm{RP})$ of the type III bursts was up to $200 \mathrm{sfu}$.

\subsection{Spikes}

First, the use of a high-pass filter allows the detection of high frequency data (including fine structures and noise). Second, we selected a quiet period and for each frequency channel ( $8 \mathrm{~ms}$ data file) we calculate the root mean square (rms) $\sigma$ of the data. Subtracting $3 \sigma$ from the high frequency data (i.e. subtraction the background noise), we are left with the data of fine structures. Then in order to determine the time of the flux maximum (necessary for determining the frequency drift rate), we fitted the time profiles by the Hermetie cubic spline method.

We define a spike according to the following selection criteria:

(1) the time duration is less than $50 \mathrm{~ms}$; 

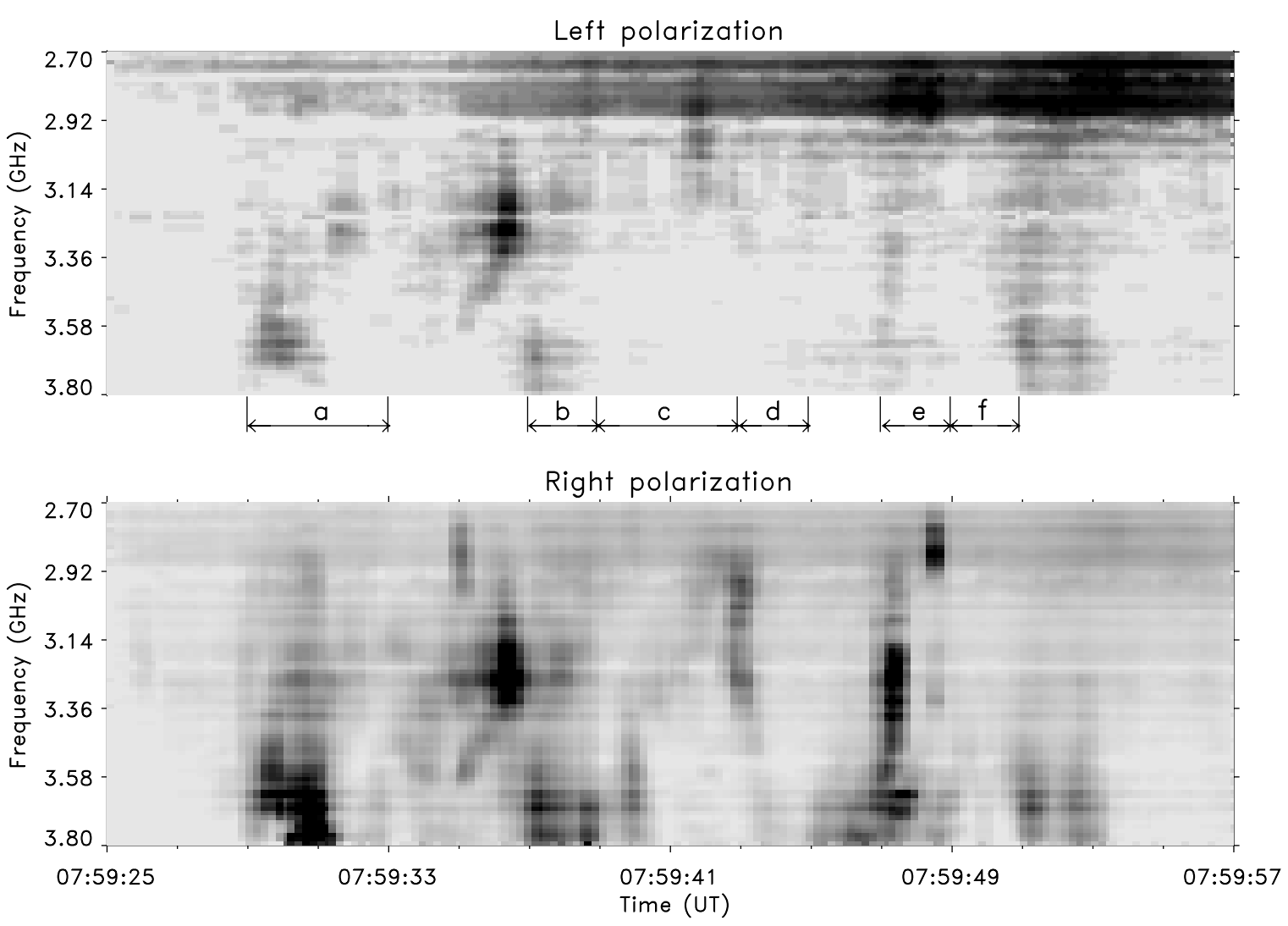

Fig. 2. Gray-scale plot of the type III bursts after eliminated the background noise in the band of $2.70-3.80 \mathrm{GHz}$ at 07:59:25-07:59:57 UT on 15 April, 1998. Enhanced flux density appears darker. The maximum flux densities are 70 sfu for LCP and $200 \mathrm{sfu}$ for RCP, and the minimum flux density is zero.

(2) the frequency bandwidth is less than $5 \%$, but has at least the coverage of $200 \mathrm{MHz}$ (a spike must exist in at least 2 channels);

(3) the peak flux is above the detection limit of $5 \sigma$.

A computer program for determining the spikes was developed following the method of Aschwanden \& Benz (1986).

Using the criteria and the computer program above, we detected more than one hundred microwave spikes at 07:59:29-07:59:50 UT. The gray-scale plots of the spikes are illustrated in Fig. 3. Statistically, the average duration of a single spike was about $27 \mathrm{~ms}$, the frequency bandwidth $2.6 \%$ of the center frequency, and the drift rate $3.07 \mathrm{GHz} / \mathrm{s}$. Both reverse (positive) drift as well as normal (negative) drift occurred in the spikes. There was no predominance of the positive or the negative sign. The flux density of LP was very weak. The polarization degree was near $100 \%$ RP. Some of the spikes were single, others were in clusters. The duration of each spike cluster was about $1 \mathrm{~s}$. The largest flux density of spikes was up to $350 \mathrm{sfu}$.

\section{Parameters of emission sources}

\subsection{Space scale and brightness temperature}

According to Zhao (1995), if we assume the magnetic field of an active region to be a dipole field under the photosphere, then the relation between the height of the radio source $(H)$ above the photosphere and its frequency $(f)$ can be estimated by

$H=d\left[\left(\frac{5.6 B_{0}(G)}{f(\mathrm{MHz})}\right)^{1 / 3}-1\right]$,

where the depth of the dipole field $d$ is about $3.5 \times 10^{4} \mathrm{~km}$, and the magnetic field strength of the photosphere $B_{0}$ can be taken to be about $1500 \mathrm{G}$. Thus the heights of the sources were $1.0 \times 10^{4} \mathrm{~km}$ to $1.6 \times 10^{4} \mathrm{~km}$, and the spatial range $(\Delta H)$ of the type III source was about $2000 \mathrm{~km}$. The spatial range of each type III burst source is estimated in Table 2. Considering that the flux density of the type III bursts was up to $200 \mathrm{sfu}$, the brightness temperature of the type III bursts was calculated to be about $2 \times 10^{10} \mathrm{~K}$.

Assuming an emission frequency depending on a characteristic frequency (such as the local plasma frequency or gyrofrequency), the upper limit $(l)$ of the spike source size could be estimated by the following relation (Benz 1986):

$$
l=\lambda \frac{\Delta f}{f},
$$

where $\lambda$ is the length corresponding to the characteristic magnetic scale, $\Delta f / f$ is the bandwidth $(2.6 \%)$ of the spikes. According to Benz (1985), we can take $\lambda=$ $6000 \mathrm{~km}$. Therefore the space scale of the spike was less 

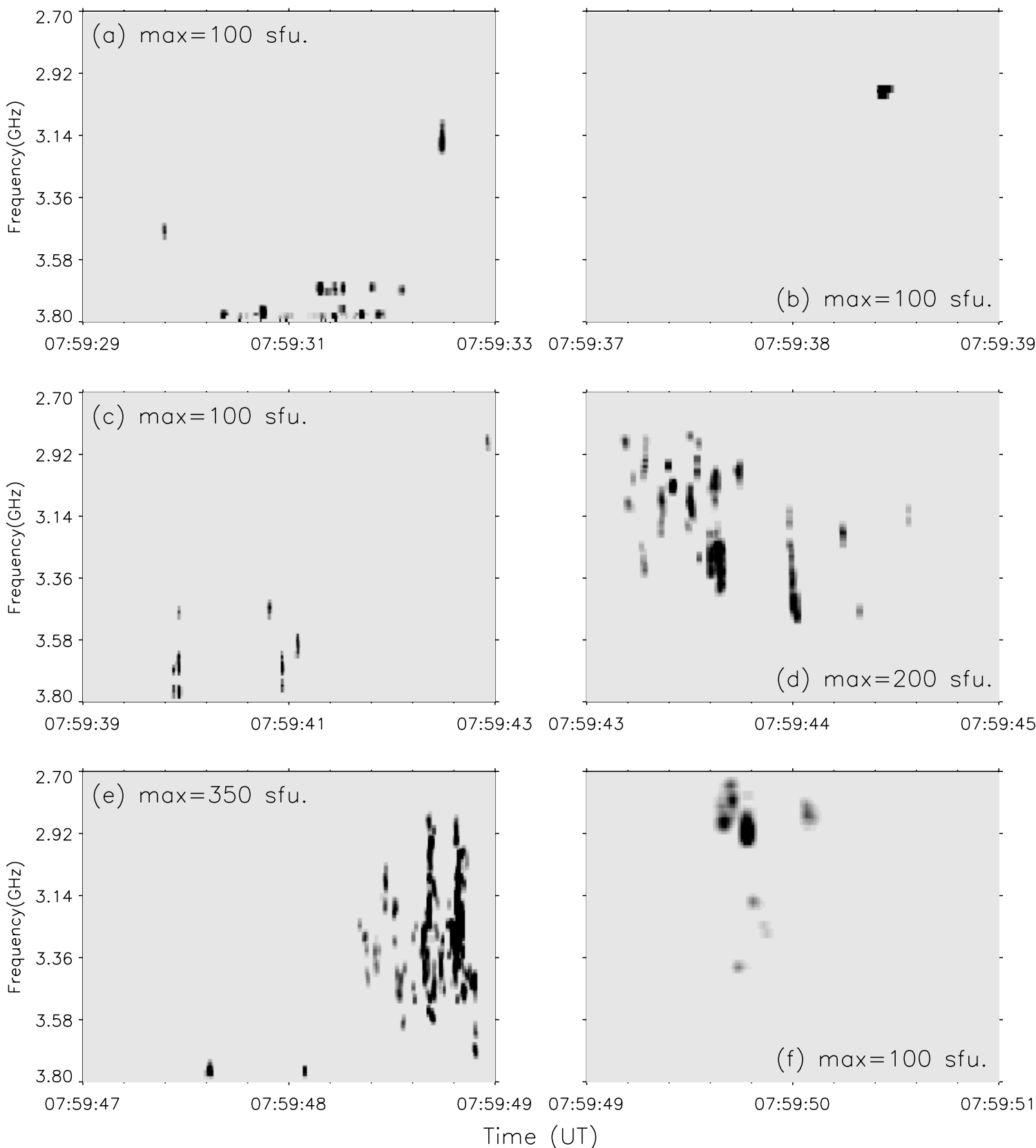

Fig. 3. Gray-scale plots of spikes in the band of 2.70-3.80 GHz at 07:59:29-07:59:50 UT on 15 April, 1998. Each cluster of spikes corresponds to the relevant periods $\mathbf{a}-\mathbf{f}$ ) marked in Fig. 2. Right circular polarization mode is illustrated. Enhanced flux density appears darker. The minimum flux density is zero.

than $150 \mathrm{~km}$. Considering the flux density of the spikes being up to $350 \mathrm{sfu}$ and the spike circular source of $150 \mathrm{~km}$, the brightness temperature of the spikes reached $10^{13} \mathrm{~K}$. This is $2-3$ orders of magnitude larger than the brightness temperature of type III bursts.

\subsection{Magnetic field strength}

It was generally accepted that type III bursts are due to fundamental or harmonic plasma emission. The plasma emission favors only O-mode emission for the fundamental frequency, so the polarization degree of the fundamental due to plasma emission may be very high (near 100\%). The polarization degree of the second harmonic depends on various conditions (Dulk 1985) and is somewhat smaller than that of the fundamental. Considering the polarization degree of $70 \%$ RP, the type III bursts should be determined as the second harmonic. Therefore, we could obtain the plasma density $n$ of $2.3 \times 10^{10}-4.5 \times 10^{10} \mathrm{~cm}^{-3}$.

In the event, the polarization degree of the spikes was very high, nearly $100 \%$, thus we also could considered the spikes as second harmonic plasma emissions. 


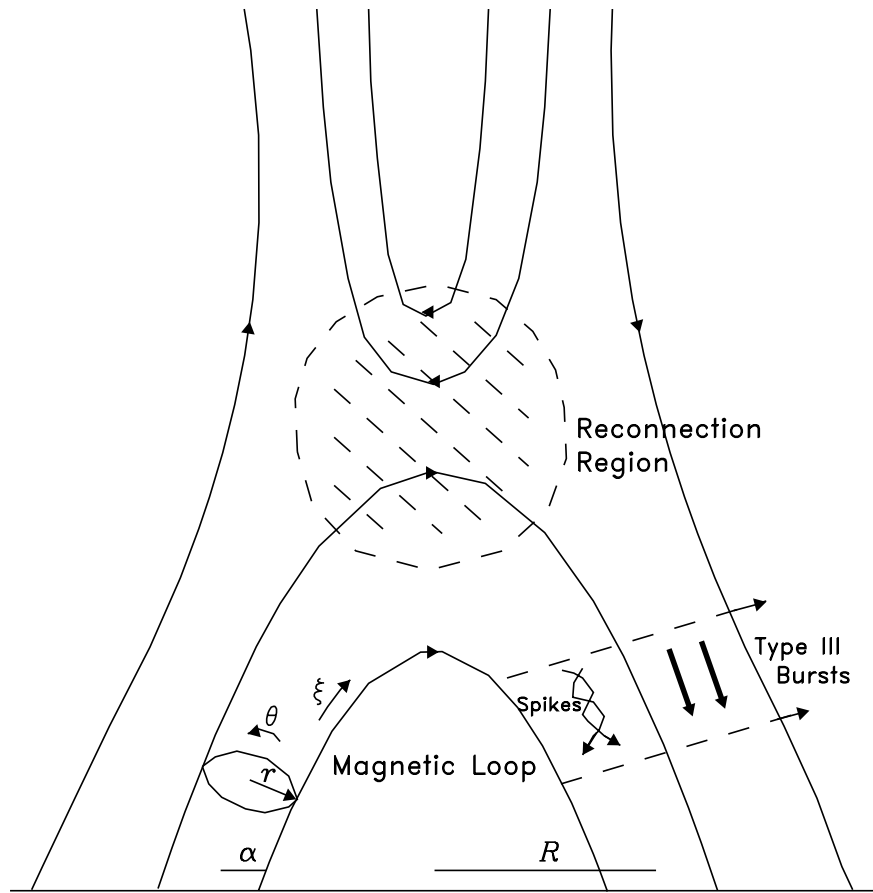

Fig. 4. Schematic drawing of the geometry of the magnetic loop in the corona. The dashed circle represents the reconnection region. $\xi, r, \theta$ are the toroidal, radial and poloidal components (the relevant magnetic components: $B_{\xi}, B_{\mathrm{r}}, B_{\theta}$ ) in the tokamak plasma loop, respectively. The transversal is perpendicular to the toroidal. $R$ refers to the toroidal (larger) radius of tokamak loop, and $\alpha$ refers to the transversal (smaller) radius. Type III bursts are caused by the energetic electrons which pour down the magnetic loop, while spikes are caused by those electrons pouring down the local complex field which results from the tearing instability.

The polarization degree $r$ of radio emissions at the second harmonic (in the sense of the O-mode) could be calculated by the following relation (Dulk 1985):

$r=\frac{85}{48} \frac{\Omega_{\mathrm{e}}}{\omega_{\mathrm{p}}}|\cos \theta| \quad\left(\Omega_{\mathrm{e}} \ll \omega_{\mathrm{p}}\right)$,

where $\theta$ is the angle between the Langmuir wave and the magnetic field. Considering the event located in N29W15 (SGD, 650-Part II, 1998), we obtained the magnetic field strength of about $300 \mathrm{G}$ for the type III burst sources and $500 \mathrm{G}$ for the spike sources. The magnetic field of the spike sources was stronger than that of the type III burst sources.

\section{Quasi-periodicity characteristics}

The quasi-periodicity of the type III bursts was about $4.5 \mathrm{~s}$ with an asymmetric and complex structure pattern. The characteristic of the asymmetric pattern could not be explained by the modulation of MHD oscillations (Aschwanden 1987). According to the study of Tajima et al. (1987), the nonlinear magnetic reconnection instability could provide some keys to understanding the quasi-periodicity - seen in this kind of type III bursts. The local magnetic field during the reconnecting stage was considered as one-dimensional. The quasi-periodicity of the type III bursts could be estimated by the following relation (Wang 1995; Tajima et al. 1987):

$\tau=2 \pi(-2 \varepsilon)^{-3 / 2} t_{\mathrm{A}}{ }^{-2}$,

where the system CGS is used. $t_{\mathrm{A}}=\lambda / v_{\mathrm{A}}$ is the Alfvén transition time, $v_{\mathrm{A}}=B / \sqrt{4 \pi n m_{i}}$. According to Tajima et al. (1987),

$\dot{a}^{2}=-2 V(a)+2 \varepsilon \quad(\varepsilon<0)$,

here $a$ is a scale factor (non-dimensional) and $\varepsilon$ is the total "energy" (dimension: $1 /$ time $^{2}$ ) in space $a$. The minimum of $\varepsilon$ is expressed as follows:

$\varepsilon_{\min }=-\frac{v_{\mathrm{A}}{ }^{4}}{2 \lambda^{2} c_{\mathrm{s}}^{2}}$,

here $c_{\mathrm{s}}=\sqrt{10 k T / 3 m_{i}}$ is the ion-acoustic velocity, $\lambda$ is the space scale of the magnetic field, taking $\lambda=6 \times 10^{8} \mathrm{~cm}$. Typically we take $B=300 \mathrm{G}$, background temperature $T=10^{6} \mathrm{~K}$, plasma density $n=10^{11} \mathrm{~cm}^{-3}$, ion mass $m_{i}=1.67 \times 10^{-24} \mathrm{~g}$, Boltzmann constant $k=$ $1.38 \times 10^{-16} \mathrm{erg} / \mathrm{K}$, then $\varepsilon_{\min }=-9.27 \mathrm{~s}^{-2}$. When taking $\varepsilon=-0.6 \mathrm{~s}^{-2}$, we obtained the quasi-periodicity $\tau=4.5 \mathrm{~s}$ according to Eq. (4).

When the total "energy" of system is $\varepsilon_{\min }$, the value of the magnetic potential energy $(V(a))$ is maximum, but the kinetic energy is zero. No magnetic potential energy can be released. However, for this event, we should take $\varepsilon=-0.6 \mathrm{~s}^{-2}\left(\sim 7 \% \varepsilon_{\min }\right)$. It suggested that more than $90 \%$ of the magnetic potential energy was transfered to kinetic energy and was released. Therefore the magnetic reconnection was a very explosive process.

The spikes often presented as very short duration (less than $50 \mathrm{~ms}$ ). This could actually be considered as some kind of quasi-periodic pulsation with a very short time scale. In order to explain an energy release process which was very explosive and released much energy during very short time scales, the resistive tearing mode instability mechanism was proposed. Waddell et al. (1979) introduced scalar functions including the magnetic scalar function $(\varphi)$ and velocity scalar function $(\psi)$ into the MHD equations to analyze the tearing instability in a tokamak plasma loop (a structure similar to the coronal loop in Fig. 4). We also used the method proposed by White et al. (1977) to regard the transversal plane $(r-\theta$ plane in Fig. 4$)$ as the rectangular coordinates $(x-y$ plane). Then considering the periodicity in component $\theta$, we assume the following relations for $\varphi$ and $\psi$ (Wang 1995):

$\varphi=\varphi_{0}(x)+\varphi_{1}(x) \cos (\mathrm{k} y) \gamma(t)$,

$\psi=\psi_{0}(x) \sin (\mathrm{k} y) \mu(t)$.

Substituting Eqs. (7) and (8) into the MHD equations of the scalar functions (Waddell et al. 1979), we can obtain (Wang 1995):

$\gamma(t) \sim \mathrm{C}_{1} \exp \left(-\frac{1}{2 t_{\mathrm{A}}} t\right) \sin \left(\frac{R}{a t_{\mathrm{A}}} t\right)$, 
$\mu(t) \sim \mathrm{C}_{2} \exp \left(-\frac{1}{2 t_{\mathrm{A}}} t\right) \cos \left(\frac{R}{a t_{\mathrm{A}}} t\right)$.

Here $\mathrm{k}$ is the space wave number, $t_{\mathrm{A}}$ is Alfvén transition time, $C_{1}$ and $C_{2}$ are parameters dependent only upon the space components $(x, y)$ and the resistivity. Therefore it is indicated that before the onset of tearing instability in a tokamak plasma loop, there is indeed a kind of very short periodicity in the poloidal turbulent magnetic field. The very short periodicity may cause the relevant change in the induced electric field, and cause the accelerated electrons to have very short periodic behaviour. Then the periodicity of the tearing instability could be estimated as follows (Wang 1995):

$\tau=\frac{2 \pi \alpha \sqrt{n \mu m_{i}}}{B_{\xi}}$

and the characteristic time $t$ of exponential growth of the tearing instability could be estimated as follows:

$t=\frac{2 R \sqrt{n \mu m_{i}}}{B_{\xi}}$

Here the SI system is used. Typically taking the background magnetic field $B_{\xi}=0.03 \mathrm{~T}, R=6 \times 10^{5} \mathrm{~m}$, $\alpha=1 \times 10^{4} \mathrm{~m}, n=1 \times 10^{17} \mathrm{~m}^{-3}, \mu=4 \pi \times 10^{-7}$ and $m_{i}=1.67 \times 10^{-27} \mathrm{~kg}$, we obtained $\tau \sim 30 \mathrm{~ms}$ and $t \sim 0.6 \mathrm{~s}$. We can find that $\tau$ corresponds to the time scale of single spike and $t$ corresponds to the time scale of the spike cluster, respectively.

\section{Conclusions}

This study contains the statistics and theoretical discussion about microwave type III bursts and the associated spikes. The major conclusions are as follows:

(1) The type III bursts and the associated spikes have very complex structures in the time profiles.

(2) The brightness temperature of the spike sources was larger than that of the type III sources.

(3) In solar corona, the magnetic field strength of the spike sources was stronger than that of the type III sources. This suggests that the magnetic field of spike sources may be more complex.

(4) Type III bursts might be caused by energetic electrons accelerated during a nonlinear reconnection process in the larger magnetic loop of solar corona. During the reconnection, the magnetic field will become more complex in local smaller area. There will be some explosive and fast instability, such as tokamak plasma instability, in the smaller area which might cause the relevant change in induced electric field, and cause the accelerated energetic electrons to excite the spikes. Our discussions were in agreement with the observation that the radio spikes appear, not due to the fragmentation of a primary energy release, but rather due to the "secondary" fragmentation, i.e. due to the presence of sufficiently strong magnetic field inhomogeneities in the coronal loop, although these inhomogeneities themselves seem to directly result from the primary energy release.

Acknowledgements. This research was supported by the National Science Foundation of China under grant No. 19773016 and No. 19973008 and by the Chinese Academy of Sciences. It also was supported by the Ministry of Science and Technology of China under grant No. G2000078403. We wish to thank Prof M. Karlický for his advice on the improvement of this paper.

\section{References}

Aschwanden, M. J. 1987, Solar Phys., 111, 113

Aschwanden, M. J., \& Benz, A. O. 1986, A\&A, 158, 102

Benz, A. O. 1985, Solar Phys., 96, 357

Benz, A. O. 1986, Solar Phys., 104, 99

Benz, A. O., \& Kane, S. R. 1986, Solar Phys., 104, 179

Dulk, G. A. 1985, ARA\&A, 23, 169

Fleǐshman, G. D., \& Mel'nikov, V. F., Physics-Uspekhi, 41, 1157

Fu, Q. J., Qin, Z. H., Ji, H. R., et al. 1995, Solar Phys., 160, 97

Karlický, M., Yan, Y. H., Fu, Q. J., et al. 2001, A\&A, 369, 1104

Tajima, T., Sakai, J., Nakajima, H., et al. 1987, ApJ, 321, 1031

Waddell, B. V., Carreras, B., \& Hicks, H. R. 1979, Phys. Fluids, 22,896

Wang, S. J. 1995, Observation and Theoretical Analysis of the Quasi-Periodic Pulsation in Solar Radio Bursts, Dissertation for Doctor's Degree

Wang, S. J., Yan, Y. H., \& Fu, Q. J. 2001, A\&A, 370, L13

Wang, S. L., Fu, Q. J., Ji, H. R., et al. 1999, Acta Astrophys. Sini., 19(3), 324

White, R. B., Monticello, D. A., et al. 1977, Phys. Rev. Lett., 39, 1618

Xie, R. X., Fu, Q. J., Wang, M., et al. 2000, Solar Phys., 197, 375

Zhao, R. Y. 1995, Ap\&SS, 234, 125 